\title{
TEST OF THE REX-RFQ AND STATUS OF THE FRONT PART OF THE REX-ISOLDE LINAC*
}

\author{
H. Bongers, S. Emhofer, D. Habs, O. Kester, K. Rudolph, T. Sieber, \\ LMU München, Garching, Germany \\ A. Schempp, C. Welsch, K. U. Kühnel, J.W.Goethe Universität, Frankfurt/Main, Germany
}

\section{Abstract}

For REX-ISOLDE (Radioactive beam EXperiments at ISOLDE / CERN) [1], a test beamline is built up at the Garching Accelerator Lab to perform $\mathrm{He}^{1+}$-experiments with the RFQ [2], the matching (rebunching) section between RFQ and IH-DT-linac, the IH-structure [3] and several electrostatic lenses of the REX-ISOLDE-mass separator [4].

In a first step, the beamline is conceived for tests with the RFQ. This paper presents the parameters and the status of the REX-RFQ, the experimental setup and the particle dynamics simulations with the COSY infinity code for beam injection and beam analysis. Furthermore it shows the design and status of the mass separator, the IHstructure and the buncher section.

\section{TEST OF THE REX-RFQ}

The REX-ISOLDE Linac consists basically of a 4-RodRFQ and an Interdigital-H Drift-Tube-accelerator, followed by three seven-gap resonators for energy variation from $0.8 \mathrm{MeV} / \mathrm{u}$ to $2.2 \mathrm{MeV} / \mathrm{u}$ [5].

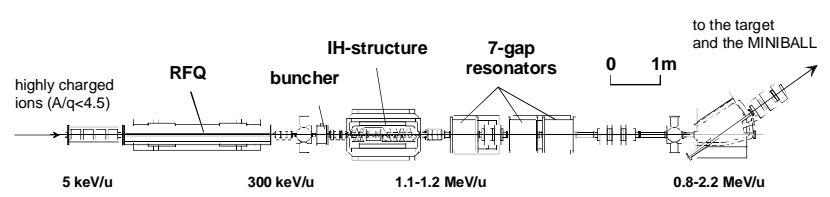

Fig. 1: The REX-ISOLDE Linac

\subsection{The RFQ}

The REX-RFQ will accelerate the radioactive ions with an A/q 3 - 4.5 from $5 \mathrm{keV} / \mathrm{u}$ to $300 \mathrm{keV} / \mathrm{u}$. The according electrode-voltage is $28-42 \mathrm{kV}$, which will require an rfpower of 30 to $40 \mathrm{~kW}$. The accelerator is similar to the GSI HLI-RFQ and to the Heidelberg High current injector RFQ. It has a total length of $3 \mathrm{~m}$ (18 stems) and operates at a resonant frequency of $101.28 \mathrm{MHz}$ (duty-cycle: $10 \%$ ). Low-level measurements of the rf-parameters were in good agreement with the design values. The measurements showed: $\mathrm{f}=101.18 \mathrm{MHz}, \mathrm{Q}=3900$ and $\mathrm{Rp}=170 \mathrm{k} \Omega \mathrm{m}$. The frequency can be shifted with two mounted piston tuners in the range $\pm 200 \mathrm{kHz}$.
The flatness was tuned from $\pm 20 \%$ to $\pm 1 \%$. The distribution of the built in tuning plates has been calculated with the code MAFIA. Fig. 2a shows the voltage distribution of a MAFIA-model with the tuning plate heigths from experimental data and unmodulated electrodes together with the measured voltage distribution of the REX-RFQ without tuning plates. The diagram illustrates the compensation of the variable capacitive load (changes in modulation and aperture) in each RFQ-cell by an appropriate variation of its inductance (different heigth of the tuning plates). Figure $2 b$ shows the tuned flatness measured with three different pertubation capacitors. The bars on the bottom of the diagram indicate the heigth of the Tuning-plates.

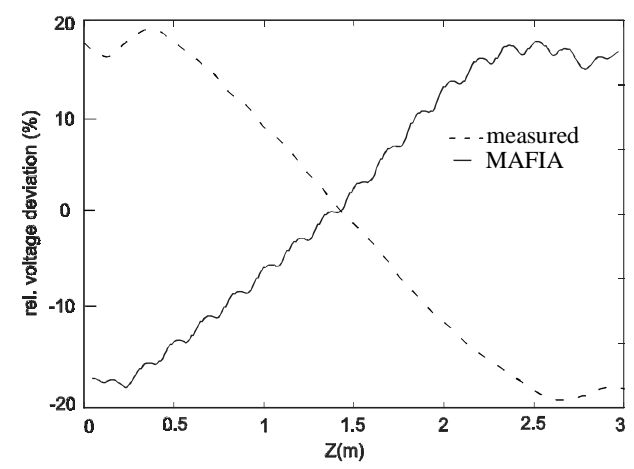

Figure 2a: MAFIA calculated and measured voltage distributions.

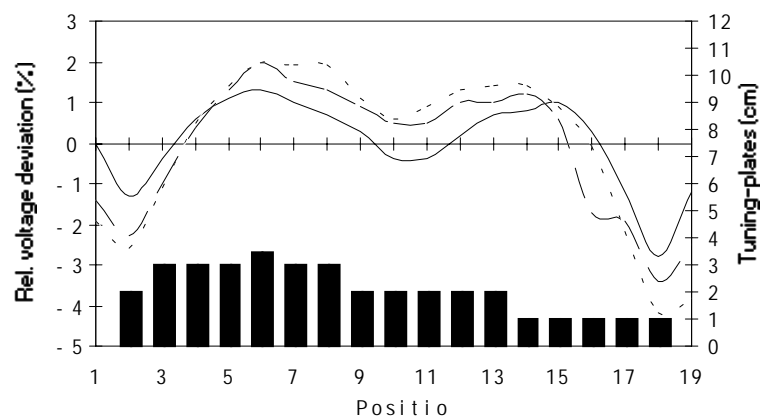

Figure 2b: Tuned voltage distribution of the REX-RFQ measured with $1.2 \mathrm{pF}$ (dotted line), $2.2 \mathrm{pF}$ (dashed line) and $3.3 \mathrm{pF}$ (solid line).

After assembly of the resonator, vacuum- and low-level rftests, the RFQ-electrodes are currently aligned with an accuracy of $1 / 10 \mathrm{~mm}$. 


\subsection{The RFQ-Test Beamline}

The test beamline for the RFQ consists of a duoplasmatron ion source, an electrostatic quadrupole quadruplet and a diagnostic box on the injection side, and an electrostatic triplet together with a $90^{\circ}$ bending magnet for beam analysis on the high energy side.

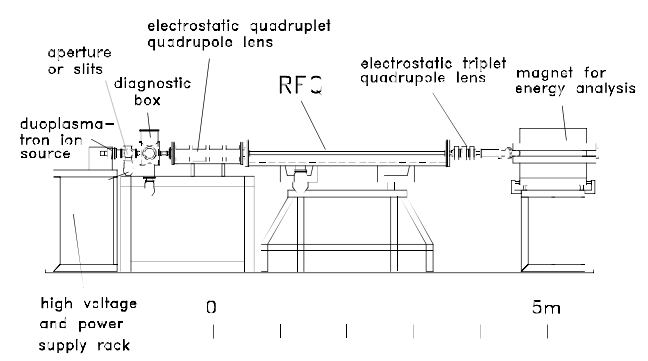

Fig. 3: RFQ test beamline.

The ion source is in operation at the source test stand of the Garching Tandem Lab. It produces a $1 \mathrm{~mA}, 20 \mathrm{keV}$ $\mathrm{He}^{1+}{ }_{\text {-beam with emittances of typically } 30-50 \pi \mathrm{mm} \text { mrad. }}$ Due to the 0-current design of the REX-ISOLDE accelerators, the current during the tests will be reduced to 1-10 $\mu \mathrm{A}$. The diagnostic box in the injection system has a Faraday cup and an aperture wheel for measurements of the beam at Munich and the pilot beam at REX-ISOLDE, as well as a low intensity device, which consists of a carbon foil with a secondary electron detection system (phosphor monitor and MSP). The upper beam current limit for this device is a pA. The detection limit is 1 ion/second. The spatial resolution for single particle detection is $0.5 \mathrm{~mm}$.
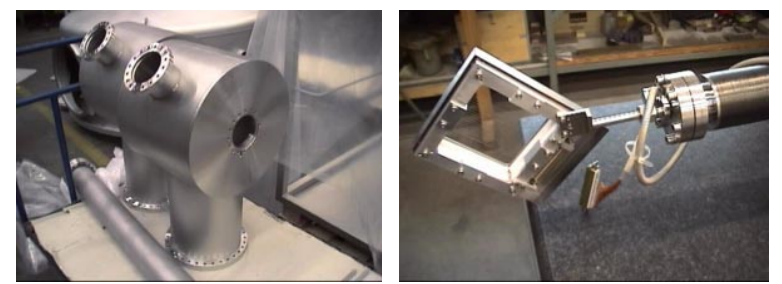

Fig. 4: Emittance measurement device designed by NTG.

The beam quality behind the source, the injection system and behind the RFQ is measured with a slit grid emittance measurement device. It is a new design of the NTG company (Gelnhausen/Germany), which moves in an angle of $45^{\circ}$ through the vertical and horizontal plane. The advantage is a measurement of both phase planes at one zposition. The resolution of this device as well as its application for different particle-energies is modified via the length of the beam tube, which connects the chambers for slit and grid. At the beam with the RFQ the angular resolution will be 0.75 (0.2) mrad for an energy of $5 \mathrm{keV} / \mathrm{u}(300 \mathrm{keV} / \mathrm{u})$ the spatial resolution is $0.2 \mathrm{~mm}$.

Beam injection and high energy beam transport have been calculated with the COSY infinity code. In the Figures 5a and $5 b$ the calculated (PARMTEQ) RFQ-acceptances and output emittances are plotted together with the calculated beam envelopes. The calculations show an acceptable matching of the beam with voltages of maximal $\pm 4 \mathrm{kV}$ in the quadrupole quadruplet (maximum voltage: $\pm 6 \mathrm{kV}$ ).
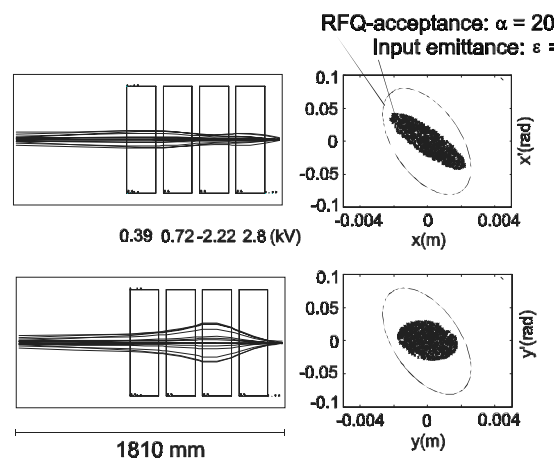

Fig. 5a: Beam injection into the RFQ

The beam transport behind the RFQ can be achieved with voltages of $\sim \pm 30 \mathrm{kV}$ in the quadrupole triplet (max. voltage: $\pm 50 \mathrm{kV}$ ). The required field strength in the $90^{\circ}$ bending magnet is $0.9 \mathrm{~T}$ (max. field: $1.2 \mathrm{~T}$ ).

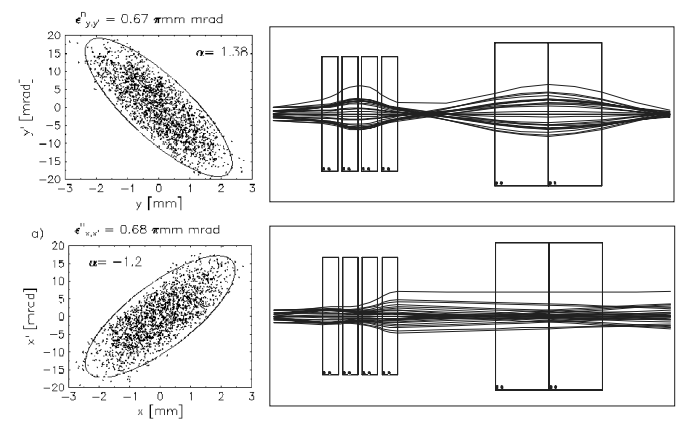

Fig. 5b: Beam transport calculated with COSY.

First beam tests will be done after the electrode alignment and the rf-conditioning of the RFQ. In the next stage, the analyzing magnet will be removed and replaced by the rebunching section and the IH-structure. Fig. 6 shows a Photo of the current setup at Garching.

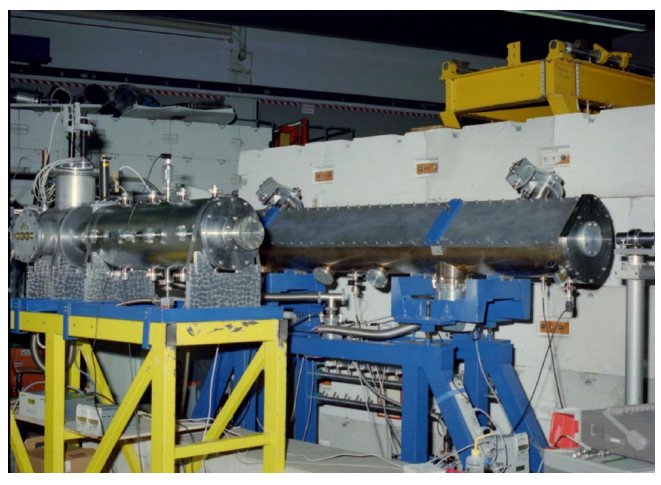

Fig. 6: Injection system (left) and RFQ. 


\section{STATUS OF THE FRONT PART OF THE REX-ISOLDE LINAC}

\subsection{The mass separator}

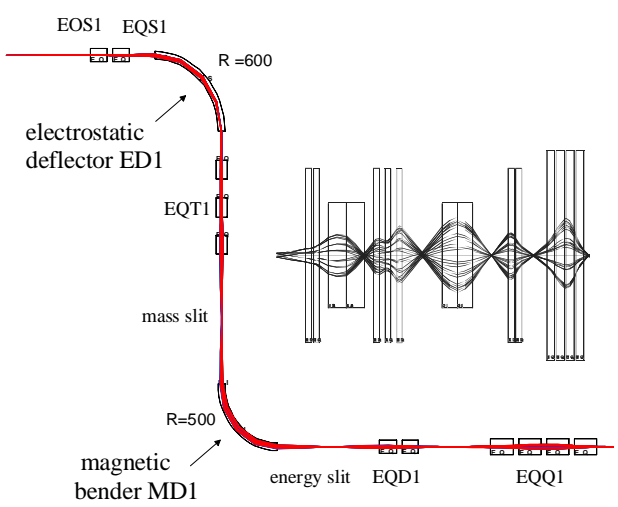

Fig. 7: Beam dynamics simulations for an achromatic system, the final design for the REX-mass separator.

The beam from the EBIS has to be mass separated to reject the ionized buffer gas atoms from the Penning- trap. The REX-ISOLDE mass separator is based on the principle of the Nier-spectrometer. The design shown in Fig. 7 was chosen in the expectation of EBIS emittances of $10 \pi \mathrm{mm}$ mrad and an energy spread of $0.25 \%$. It has a resolution of 150 , which is sufficient to separate most of the radioactive ions from the buffer gas ions (f. ex. not ${ }^{40} \mathrm{Ar}$ from $\left.{ }^{40} \mathrm{Ka}\right)$.

Currently, the electrostatic octupoles and quadrupoles as well as the electrostatic deflector are in production at our mechanical workshop, while the magnet is manufactured by Sigma Phi (Vannes/France)

\subsection{The matching section}

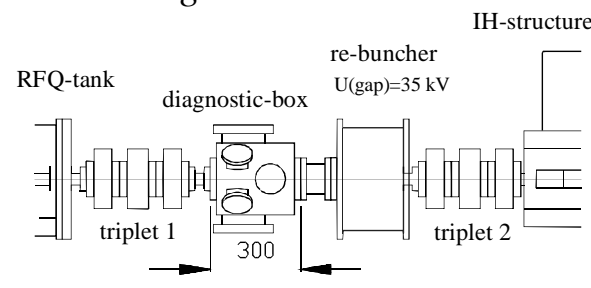

Figure 8: Matching section for the IH-structure.

The Buncher for the matching section is a 3-gap splitring resonator with a gap voltage of $35 \mathrm{kV}(\mathrm{A} / \mathrm{q}=4.5)$. It was built at the IAP/Frankfurt. The structure is completely assembled and will be delivered to Munich after the rfpower tests. The magnetic lenses are ordered at DANFYSIK (Jyllingen/Denmark) and will be delivered in May. The whole Linac has been calculated with the codes TRANSPORT and COSY for different EBIS emittances. The calculations show required field gradients of the magnetic quadrupoles in the matching section up to 60 $\mathrm{T} / \mathrm{m}$.

\subsection{The IH-structure}

The REX-IH structure is like the CERN lead injector and the GSI HLI IH-Linac a 0-phase synchronous particle structure excited in the $\mathrm{H}_{110}$-Mode. It has a length of 1.5 $\mathrm{m}$ and a diameter of $0.6 \mathrm{~m}$. The overall accelerating voltage is $4.2 \mathrm{MV}$ (20 gaps) for ions with $\mathrm{A} / \mathrm{q}=4.5$ at a required rf-power of $60 \mathrm{~kW}$.

The end energy can be varied from $1.1 \mathrm{MeV} / \mathrm{u}$ to 1.2 $\mathrm{MeV} / \mathrm{u}$, whereby this variation is achieved with capacitive plungers, which modify the voltage distribution in the gaps. Fig. 9 shows photos of the IH-structure during manufacturing at NTG. It also shows the measured, MAFIA-calculated and design-voltage distributions of a 1:2 model for two different end energies together with the corresponding positions of the capacitive plungers.

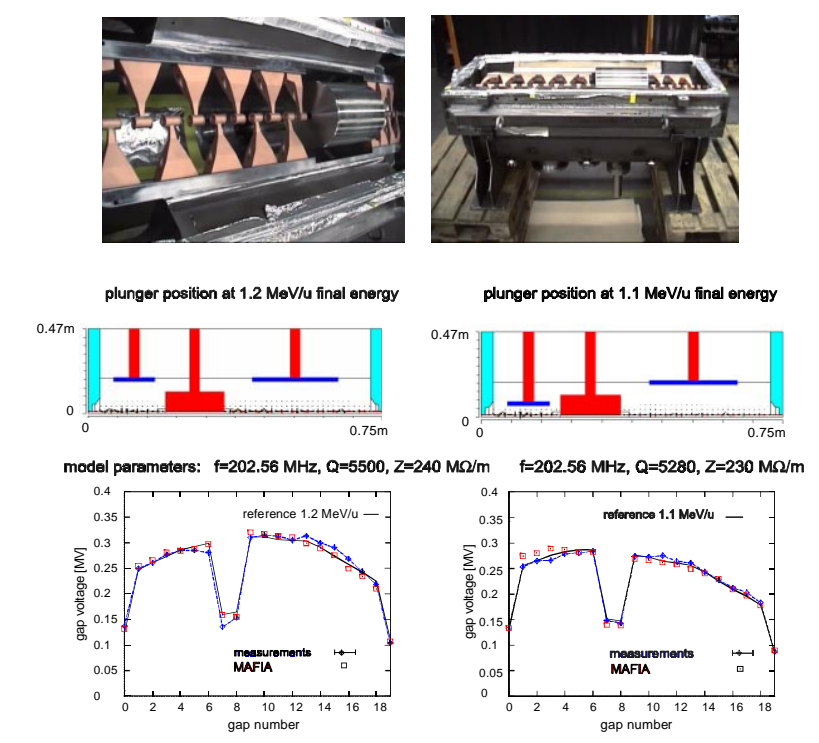

Fig. 9: Drifttube structure and tank of the REX-IHaccelerator (above). Calculated and measured voltage distributions in a 1:2 model.

The IH structure is presently copper plated at GSI and will be integrated in the test beamline after power tests at Munich. The status of the 7-gap resonators is presented at this conference.

\section{REFERENCES}

[1] D. Habs et al., "The REX-ISOLDE Project", NIM B 139 (1998) 128

[2] T. Sieber et al., „Design and status of the REXISOLDE RFQ“, Proc. LINAC '98, Aug. 1998 Chicago

[3] O. Kester et al., "The Status of the REX-ISOLDE project”, Proc. of the CAARI '98, Denton, Nov. 1998.

[4] R. Rao et al., NIM A 427 (1999) 170

[5] R. von Hahn., this conference

* work supported by the BMBF under contract 06 LM 868 I/TP:4 